\title{
INMIGRACIÓN Y NECESIDADES FORMATIVAS \\ DE LOS CUIDADORES
}

\author{
Manuel Moreno Preciado \\ Enfermero y antropólogo. Profesor del Departamento de Enfermería \\ de la Universidad Europea de Madrid \\ Teresa Martín Hernández
}

Enfermera y psicóloga. Subdirectora de Enfermería del Hospital Virgen de la Torre de Madrid

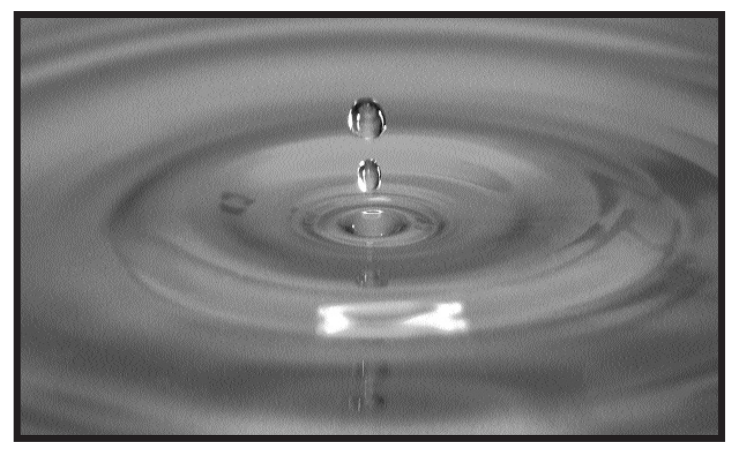

\section{INMIGRATION AND TRAINING NEEDS FOR CARERS}

\section{ABSTRACT}

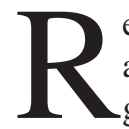

ecent studies show a lack of knowledge about the cultures of different ethnic groups in the Spanish setting. It has been largely recognized that several cultural issues such as beliefs, myths, religion, tabooes, habits etc, have an effect in the health-disease-care process.

This article shows the need for education programmes in cultural diversity as an essential tool for this new situation, which nowadays has become a challenge for health professionals and particularly for the nursing profession. Studies related to the provision of care to different ethnic groups are identified and analized in this article. A study of the present knowledge of nursing professionals with respect to cultural diversity, through the different educational programmes offered in various institutions, shows the lack of preparation in these professionals to cope with present needs and expectations.

Key words: Training, integration, migration, transcultural care

\section{RESUMEN}

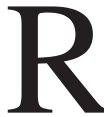

ecientes estudios muestran un gran desconocimiento de los españoles sobre las culturas de origen de los inmigrantes. Los elementos de la cultura, como los mitos, la religión, los tabúes, los usos y costumbres, etc, inciden en el proceso salud/enfermedad/atención. Por otro lado el inmigrante es una persona especialmente vulnerable debido a las condiciones de precariedad y desarraigo que acompañan al hecho migratorio, sobretodo en su primera fase.

Este artículo plantea la necesidad de la formación en diversidad cultural como herramienta imprescindible para que los cuidadores puedan abordar con éxito esta nueva situación, que supone un reto para todos los profesionales de la salud y particularmente para los enfermeros. Se exponen y analizan varios estudios en relación al contexto de la atención sanitaria a la población inmigrada. Se presenta y analiza un estudio sobre el estado actual de la formación de los profesionales de enfermería en diversidad cultural, a través de los diferentes programas de formación continua ofertados desde los centros asistenciales y desde otras instituciones y se llega a la conclusión de que la formación transcultural de los profesionales de enfermería es insuficiente para las necesidades y expectativas actuales.

Palabras clave: formación, integración, migración, cuidado transcultural.

\section{INTRODUCCIÓN Y OBJETIVOS}

Los inmigrantes se sitúan en estos momentos en el centro del debate político, social y cultural en España. Los españoles muestran perplejidad y sorpresa ante esta inmigración, ante un hecho que se 
vive con preocupación (Izquierdo, 1996) Esta sorpresa que revelan recientes estudios, tiene su fundamento en lo inesperado del fenómeno. No hace muchos años éramos un país de emigrantes y ahora son otros los que vienen a España a buscar una nueva vida. Y esta sorpresa se traduce en perplejidad y en interrogantes: ¿esto qué significa?, ¿hasta donde puede llegar?, ¿Qué consecuencias tendrá para las formas de vida colectivas?, etc. Los medios de comunicación reflejan a diario los problemas y dilemas que representa la convivencia intercultural (Moreno Preciado, 2002).

Recientes estudios muestran un gran desconocimiento de los españoles sobre las culturas de origen de los inmigrantes. Los inmigrantes refieren importantes dificultades de adaptación a nuestro entorno (Criado, 2001) Todo parece indicar que estas dificultades tienen también su continuidad en el marco de la asistencia sanitaria. Es indudable que los elementos de la cultura, como los mitos, la religión, los tabúes, los usos y costumbres, etc, inciden en el proceso salud/enfermedad/atención (Roca i Capara, 2001).

La enfermedad y la hospitalización son momentos transcendentales marcados por la fragilidad física y psíquica de las personas y por la presencia de un entorno distinto que obligará al paciente a un importante esfuerzo de adaptación. En el caso de los inmigrantes estos cambios son aún mayores, por lo que tendrán que hacer uso con mayor profusión de sus mecanismos de adaptación.

Por otro lado el inmigrante es una persona especialmente vulnerable debido a las condiciones de precariedad y desarraigo que acompañan al hecho migratorio, sobre todo en una primera etapa. La inmigración que conocemos hoy está fuertemente marcada por la situación ilegal de muchos migrantes; por condiciones de vida y de trabajo muy deterioradas que tienen una incidencia importante en la salud.

Este trabajo tiene como objetivo plantear la situación de la formación de los profesionales de enfermería en relación a los cuidados de salud a la población inmigrada y la repercusión de la misma en la práctica cuidadora, considerando la formación transcultural como una herramienta imprescindible para que los cuidadores puedan abordar con éxito esta nueva situación, que supone un reto para todos los profesionales de la salud y particularmente para los enfermeros.

\section{MATERIAL Y MÉTODOS}

Vamos a exponer brevemente los principales elementos de este trabajo, a través de una mirada enfermera enfocada en dos planos:

1. Una visión de conjunto sobre la situación actual de la atención sanitaria y de enfermería a los inmigrantes, en base al estado de investigaciones actuales.

2. La situación actual de los programas de formación en diversidad cultural ofertados a los profesionales de enfermería en la Comunidad de Madrid.

\section{RESULTADOS \\ 1. Una visión de conjunto}

En la bibliografía española hay poca evidencia sobre las migraciones y el proceso salud/enfermedad/atención (Mascarella, Comelles, Allué) que quizás obedezca a la relativa novedad del fenómeno. No obstante en el colectivo enfermero es sujeto de interés y gran actualidad. En el III Congresos sobre inmigración en España, celebrado el mes de Noviembre de 2002 en Granada se presentaron numerosos trabajos sobre el tema presentados por enfermeras/os, y lo mismo puede decirse de las II Jornadas sobre Cultura de los Cuidados celebradas también en Noviembre de 2002 en Alicante. En Junio de 2003 se ha celebrado en Zaragoza la I Reunión de Enfermeras y Enfermeros Antropólogos, con el lema de "Cuidados de enfermería en una sociedad multicultural" donde el eje principal ha sido la atención a la población inmigrada. Las revistas científicas de enfermería dedican cada día más espacios al tema, no sólo las de carácter más generalista, sino también las especializadas.

¿Cuáles son los centros de interés sobre los inmigrantes para nuestro colectivo profesional? Las áreas donde hay mayor interés por el fenómeno de la inmigración se sitúa en primer lugar en aquellos profesionales relacionados con programas de Cooperación Internacional, Redes de Solidaridad, Ayuda Mutua e Intervenciones de Urgencia, y también en los/as enfermeros/as que desarrollan su actividad en zonas con abundante 
presencia de inmigrantes. De forma general en los procesos asistenciales la preocupación del profesional de enfermería está en cómo adaptar el cuidado a los patrones culturales de los inmigrantes. En los trabajos aludidos se destaca que el inmigrante requiere de los cuidadores mayor atención a aspectos que también son referidos por el conjunto de usuarios, como el trato, la información, etc. Los aspectos donde los inmigrantes encuentran mayor dificultad de adaptación, o que producen mayor insatisfacción y rechazo son aquellos relacionados con la higiene, la alimentación, la intimidad y la comunicación.

Hemos querido, también, en este trabajo hacer una aproximación al estado del cuidado transcultural (Leinninger, 1999) en el marco de la formación universitaria. ¿Cuál es el estado actual de la formación universitaria de los futuros enfermeros en relación al cuidado transcultural? ¿Qué piensan los estudiantes en relación a este problema?

En un estudio realizado entre alumnos de enfermería en la Universidad Europea de Madrid sobre la percepción del cuidado a los pacientes inmigrantes se obtienen los siguientes resultados:

1. Para los estudiantes el estado de salud de los inmigrantes es peor que el de los autóctonos.

2. Su cuidado es más complejo y problemático.

3. Se desconocen en gran medida sus hábitos y costumbres, pero sin embargo éstos se valoran como negativos para el mantenimiento de la salud.

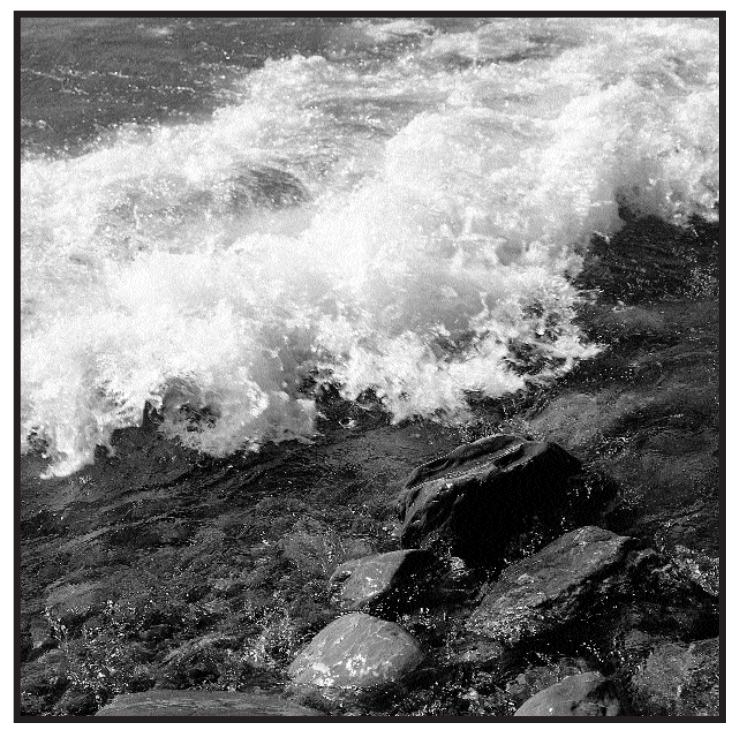

4. Es necesaria la formación transcultural de los estudiantes y profesionales, pero más aún la educación sanitaria a los inmigrantes.

Del análisis de este estudio destacaremos que la opinión de los estudiantes, a veces contradictoria, es similar en sus apreciaciones a la reflejada en los estudios de percepción del personal sanitario, que a su vez no difiere en mucho del estado de opinión general existente en la población española sobre la inmigración.

El interés que actualmente despierta entre los enfermeros y enfermeras el cuidado hacia los inmigrantes no parece estar acompañado de la misma intensidad en la formación universitaria, pues son pocos los programas específicos dirigidos a los futuros profesionales. Una revisión a través de las páginas Web de las diferentes universidades españolas donde se cursan estudios de enfermería muestra que en la mitad de ellas no se contempla ninguna asignatura especifica sobre diversidad cultural. Entre las que si lo tienen, en la gran mayoría son asignaturas optativas (40\%), de entre 4 y 5 créditos. Una minoría tienen asignaturas obligatorias (7\%) y/o de libre elección (3\%).

Muchas de estas asignaturas son de reciente creación, lo que hace pensar que también la universidad va incorporando esta preocupación a los planes de estudios.

\section{Los programas de formación para profesio- nales en la Comunidad de Madrid}

Para conocer los programas formativos sobre diversidad cultural a los cuales pueden acceder los enfermeros y las enfermeras de la Comunidad de Madrid, hemos realizado una revisión de los programas de formación realizados y/o programados en los años 2002/2003 en los siguientes ámbitos:

- Programas de formación continua en las diferentes Áreas de Salud, tanto en Primaria, como en Especializada.

- Programas de Formación impartido por instituciones como Consejería de Salud, Escuela Nacional de Sanidad, Consejo General de Enfermería, Colegio de Enfermería de Madrid y sindicatos.

De esta revisión hemos obtenido los siguientes resultados: 


\subsection{Atención Primaria:}

- Área 1: Una Jornada: "Inmigración y Salud".

- Área 2: Una Jornada: “Cuidando en la diversidad". Un Curso sobre "Inmigración".

- Área 5: Dos cursos sobre "Atención al niño inmigrante" (20 horas).

- Área 6: Dos cursos sobre "Inmigración y salud".

- Área 8: Un curso sobre "Atención sociosanitaria al paciente inmigrante" (20 horas).

- Área 9: Dos cursos sobre "Atención al inmigrante" (30 horas). Una Jornada sobre "Inmigración" (realizada en 3 Centros de Salud).

- Área 10: Un curso sobre "Salud e inmigración". (10 horas).

- Área 11: Dos cursos sobre "La inmigración y sus repercusiones sobre los sistemas de salud"

2.2. Atención Especializada: sobre 15 hospitales públicos estudiados no se ha encontrado ningún curso realizado o programado, y se han realizado dos Jornadas sobre diversidad cultural (hospitales Santa Cristina y Severo Ochoa). Hay que reseñar que algunos de los cursos organizados desde las Áreas de Atención Primaria, se han hecho en colaboración con Atención Especializada, por lo que han podido asistir enfermeras/os de los hospitales de referencia.

\subsection{Otras instituciones:}

Organización colegial: El Colegio de Enfermería de Madrid tiene programados 3 cursos relacionados con la inmigración:

a) Curso superior sobre el entorno jurídico del extranjero en España. (300 horas)

b) Curso sobre promoción de la salud en la población inmigrante subsahariana. (18 horas)

c) Curso sobre las representaciones sociales y culturales de la salud y la enfermedad en las sociedades en desarrollo. (18 horas)

Administración regional: ningún programa ofertado.

Escuela Nacional de Sanidad: Un curso sobre "Promoción de la salud de la población inmigrante. (30 horas).

Organizaciones sindicales: Un curso sobre "Atención intercultural de salud para profesionales de enfermería. (12 horas) Fuden/Satse.

Los contenidos de los diferentes programas son de índole diverso, donde en algunos se trata direc-

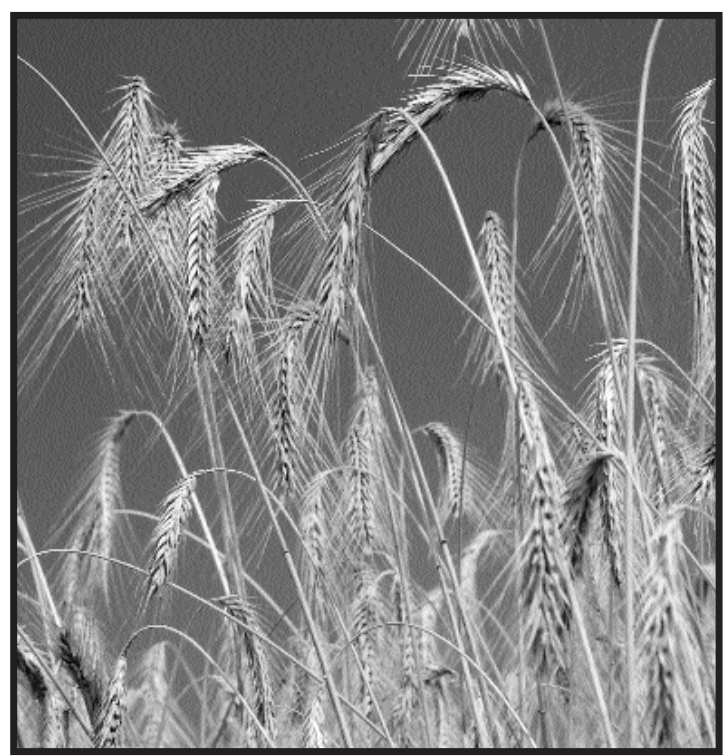

tamente el cuidado a la población inmigrante y en otros se refieren a aspectos más generales, cómo el estudio de la marginalidad, la pobreza, la diversidad cultural, etc. Se trata, con una sola excepción de cursos de corta duración.

Podemos decir que los programas sobre diversidad cultural a los cuales tienen acceso los enfermeros en la Comunidad de Madrid, son escasos en cualquiera de los ámbitos estudiados, pero muy especialmente en Atención Especializada. De aquí que nos interroguemos por las causas: ¿por qué este desfase entre el interés creciente en el colectivo enfermero y la escasa repercusión en el ámbito de la formación? No tenemos respuesta para esta pregunta. Quizás podamos barajar la hipótesis de que el interés concretado en congresos, jornadas y artículos científicos obedezca al número importante de enfermeras y enfermeros que han cursado estudios de Antropología Social y Cultural en los últimos años. Y también que el tema no se considere una prioridad, por el momento, entre quienes promueven y organizan la formación continua de los profesionales de enfermería. No obstante de los contactos establecidos con los responsables de formación cabe destacar que muchos de ellos tienen in mente poner en marcha algún programa de este tipo, pues consideran que hay una demanda formativa, y que están trabajando sobre la forma más idónea de plantear estos programas. 


\section{CONCLUSIONES Y DISCUSIÓN}

La creciente llegada de personas de diferentes culturas supone para los cuidadores un reto importante. Se requiere de nosotros la atención a nuevas demandas en contextos más complejos, donde se hace necesario conocer otras formas de entender el proceso salud/enfermedad/atención, así como entender las repercusiones del hecho migratorio en la salud de los inmigrantes. Es preciso entender la migración como un proceso liminal (Van Gennep, 1986) con sus ritos de paso, y con problemas de salud asociados a cada una de sus fases. Hacer frente a esta complejidad requiere asumir una práctica profesional centrada en la aplicación de unos cuidados integrales y holísticos, desmarcada del modelo biomédico. A este fin se hace necesaria la profundización en la formación transcultural de los cuidadores de la salud, que debe concretarse en la puesta en marcha de programas formativos específicos, como los anteriormente descritos en relación a la problemática de la inmigración, pero también se requiere que la filosofía del cuidado transcultural impregne los contenidos de los programas de formación general.

\section{BIBLIOGRAFÍA}

- CRIADO, M.J. (2001) La línea quebrada. Historias de vida de migrantes. Cees, Madrid.

- IZQUIERDO, A. (1996) La inmigración inesperada: la población extranjera en España (1991-1995). Trotta, Barcelona.

- LEININGER M. (1999) Cuidar a los que son de culturas diferentes requiere el conocimiento y las aptitudes de la enfermería transcultural. Cultura de los Cuidados 6, 5-8.

- MASCARELLA L, COMELLES JM, ALLUÉ X, (2002) La percepción en los colectivos inmigrantes no comunitarios de los dispositivos de salud en España, [Documento en línea]. <http://www.salutepertutti.org /inglese/immi_say/spa/2.4.2\%20sp.\%20I1\%20caso\%20 spagnolo.pdf $>$ [consulta: 19-11-2002].

- MORENO PRECIADO, M. (2002) Fronteras culturales de ayer y de hoy: aprendamos del pasado. Gazeta de Antropología, ${ }^{\circ} 18$.

- ROCA I CAPARÀ, N. (2001) Inmigración y salud. Situación actual en España, Rol de Enfermería, volumen 24 (11), 56-62.

- VAN GENNEP, A. (1986) Los ritos de paso. Taurus, Madrid.

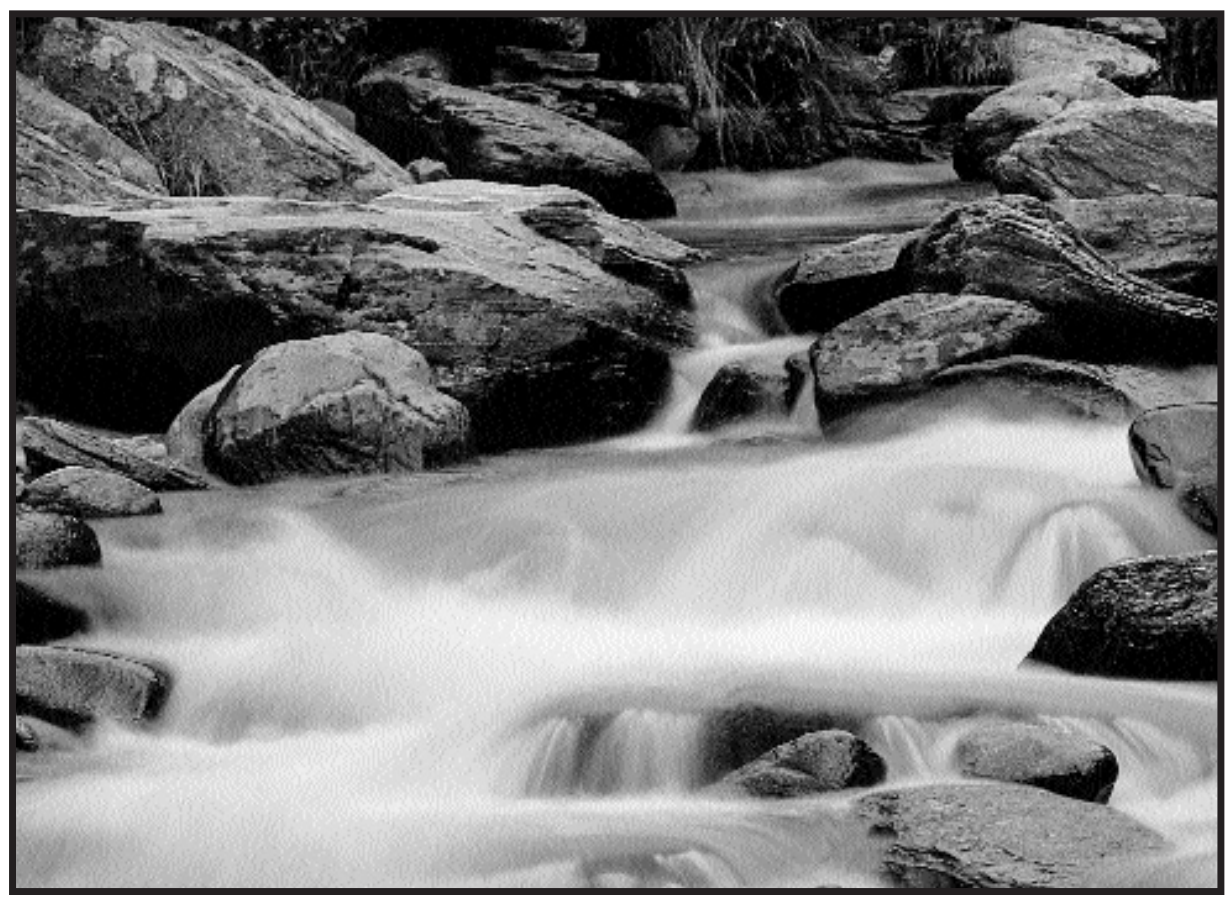

